\title{
Documentation générale
}

\section{COMMENT SE RĖGLENT EN RÉGION ÉQUATORIALE LES PROBLÈMES BIOLOGIQUES PROPRES A CHAQUE HÉMISPHËRE ?}

\author{
par G. THIERY
}

Il est classiquement admis que les périodes de la vie génıtale de la plupart des animaux sont sous la dépendance de la saison locale. Par exemple les chaleurs des équidés sont printanières tant dans l'hèmisphère nord que dans l'hémisphère sud, c'est-ả-dire qu'elles sont séparées par une période de 6 mois correspondant au décalage des saisons. Que se passe-t-il à la jonction des deux hémisphères, c'est-à-dıre sous l'èquateur ? Le problème s'est posé à nous au cours d'une mission en zone équatoriale du Brésil, à propos du renard.

Etudiant les possibilités de transmission de la rage par le renard dans la région de Fortaleza (état du Ceará), à environ $4^{\circ}$ de latitude sud, il était important de connaître les diverses époques de la vie génitale de cet anımal. Nous savions qu'en Europe ou en Amérique du Nord les chaleurs du renard ont lieu en janvier-février. En toute logique dans l'Amérique du Sud elles doivent se situer en jullet-août. De faıt des paysans dignes de for nous ont affirmé avoir par 2 fois, au mois d'août, tué des renards accouplés. Pıégeant les renards en brousse au cours des mois d'août et septembre, nous fûmes surpris de constater à l'autopsie des premières femelles capturées que les ovaires étaient au repos complet. Fallait-il admettre que les femelles gestantes puis allaitantes sortant peu du terrier ne se faisaient pas capturer ? Ma1s même dans ce cas on devait considérer qu'ıl exıstait đes femelles à vie génitale interrompue. La réponse à ce problème fut apportée assez rapidement par la capture d'un jeune âgé de 5 à 6 mois. Sa mère avalt donc eu ses chaleurs en janvier ou février. Dès lors nous nous trouvons en présence d'une population vulpine dont une partie des individus a ses chaleurs en janvier-février et l'autre en juillet-août.

Ceci ne devrait pas surprendre car la végétation équatoriale nous montre le même phénomène. C'est ainsi que le flamboyant qui fleurit à Dakar en juin-jullet et au sud du Brésil en décembre-janvier montre dans la région de Fortaleza deux périodes de floraisons : une importante à la fin de l'année et une petite en juin-juillet. Il en est de même pour le tulipier et d'autres végétaux.

Les physiciens ont l'habitude de nous dıre que l'eau quı s'écoule lıbrement par un trou régulier situé au fond d'un récipient forme un tourbillon dont le sens diffère selon l'hémisphère. Sous l'équateur ou bien le tourbillon ne se forme pas ou bien son sens est varıable. On peut rattacher à ce phénomène la tendance de certaines plantes grimpantes (haricot, houblon, etc.) à s'enrouler toujours dans le même sens autour d'un support et à se dérouler lorsqu'artificiellement on les a mises dans la mauvaise position. Dans le Ceara il est un palmier : le Carnauba, dont l'insertion des feuilles sur le tronc dessine une hélice. Comme les feuilles sont récupérées par l'industrie pour en extraire une cire, le tronc des palmiers porte pendant longtemps la base des feuilles qui dessine des spirales. Là encore il existe deux populations de palmiers ceux dont les spirales tournent dans un sens et ceux dont les spirales tournent dans l'autre. Deux palmiers voisins peuvent présenter des spirales opposées. En première approxımation les deux populations sont équivalentes.

Loin de nous l'idée d'une généralisation qui pourrait donner lieu à des interprétations pour le moins fanta1sistes, telles que l'extrapolation à la spirale des acides nucléiques.

Ainsi il semble que la nature a1t résolu le passage d'un hémisphère ả l'autre par la juxtaposition dans la zone équatoriale de 2 groupes de population adaptés à l'un ou l'autre hémisphère qu'il s'agisse de phénomènes liés à la rotation de la terre autour du soleil (cycle de reproductıon des animaux et des végétaux) ou de la rotation de la terre sur ellemême (spiralisation chez les végétaux). 